Ilyas Uyanik ${ }^{1}$, Ashik Khatri ${ }^{1}$, Dinesh Majeti ${ }^{1}$, Muhsin Ugur ${ }^{1}$, Dvijesh Shastri ${ }^{2}$ and loannis Pavlidis ${ }^{1}$

${ }^{1}$ Computational Physiology Laboratory, University of Houston, Houston, TX 77004, ${ }^{2}$ Computer Science, University of Houston - Downtown, Houston, TX 77002 iuyanik@uh.edu, arkhatri@uh.edu,dmajeti@uh.edu,mugur@uh.edu, shastrid@uhd.edu, ipavlidis@uh.edu

Introduction

Recent developments in mobile computing have enabled ubiquitous monitoring of walking activity via smartphone accelerometers.

\section{5}

W. Walking apps map accelerometer values to caloric values through a calibration algorithm.

\section{5}

These calibration algorithms assume a flat surface, which is not always true and can introduce significant errors.

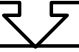

\begin{tabular}{|c|c|c|}
\hline We outline a novel calibration method that takes into account inclination, \\
thus, improving the estimation in mobile walking apps.
\end{tabular}

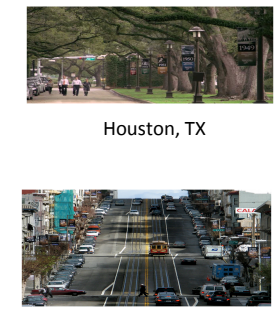

San Francisco, CA
Data Analysis

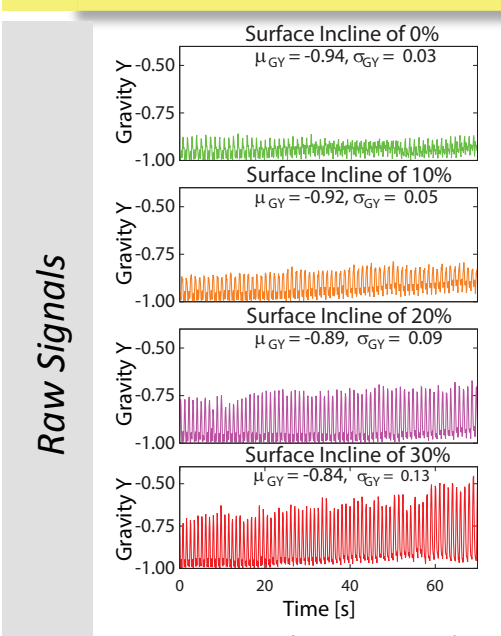

$Y$-axis Gravity values from walking sessions at $3 \mathrm{mph}$.

\section{Summary}

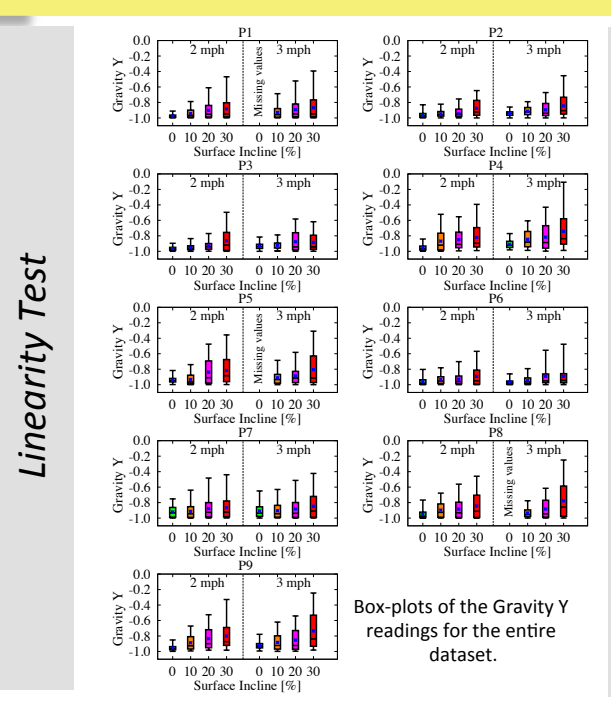

\title{
Experimental Design
}
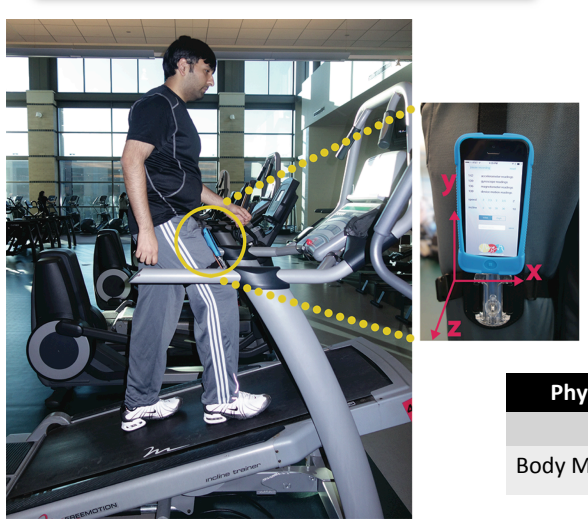

$-n=9$ (4 normal weight, 4 overweight, 1 obese )

-8 experimental phases ( 2 speeds $X 4$ inclines)

-Speed: $2 \mathrm{mph}$ and $3 \mathrm{mph}$

- Surface incline: $0 \%, 10 \%, 20 \%$, and $30 \%$

- Accelerometer data was collected only during the exercise sessions.

\begin{tabular}{|ccc|}
\hline Physical Attribute & $\boldsymbol{\mu} \pm \boldsymbol{\sigma}$ & Range \\
\hline Age $(\mathrm{yr})$ & $26.77 \pm 3.59$ & $23-34$ \\
\hline Body Mass Index $\left(\mathrm{kg} \cdot \mathrm{m}^{-2}\right)$ & $25.95 \pm 3.30$ & $22.1-32.7$ \\
\hline
\end{tabular}

\begin{tabular}{|r|c|c|c|} 
Surface Incline & $\mathbf{0} \%$ & $\mathbf{1 0} \%$ & $\mathbf{2 0} \%$ \\
\hline
\end{tabular} \begin{tabular}{|c|c|c|c|}
\hline $0 \%$ & - & - & - \\
\hline $10 \%$ & 0.0080 & - & - \\
\hline $20 \%$ & 0.0023 & 0.004 & - \\
\hline $30 \%$ & 0.0001 & 0.0000 & 0.0006 \\
\hline
\end{tabular}

Paired t-test results (P values) for the $2 \mathrm{mph}$ walking sessions; $n=9$ fo all tests. \begin{tabular}{|c|c|c|c|}
\hline Surface Incline & $\mathbf{0} \%$ & $\mathbf{1 0} \%$ & $\mathbf{2 0} \%$ \\
\hline $0 \%$ & - & - & - \\
\hline $10 \%$ & 0.0311 & - & - \\
\hline $20 \%$ & 0.0003 & 0.0000 & - \\
\hline & 0.0008 & 0.00 & 0.030 \\
\hline
\end{tabular} \begin{tabular}{|c|c|c|c|}
\hline $20 \%$ & 0.0003 & 0.0000 & - \\
\hline $30 \%$ & 0.0008 & 0.0001 & 0.0130 \\
\hline
\end{tabular}

Paired t-test results ( $P$ values) for the 3 mph walking sessions; $n=9$ for all tests except for the $0 \%-10 \%$ pair for which $n=6$.

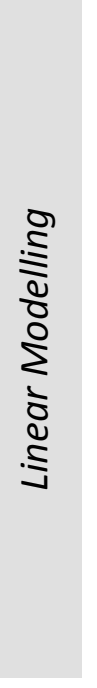

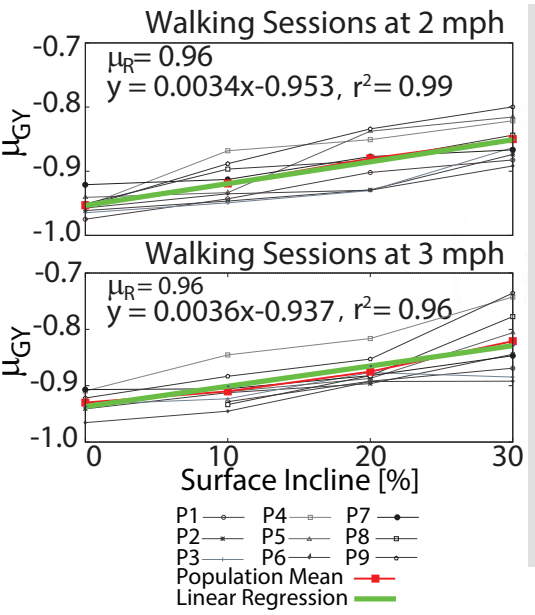

Once surface incline is estimated from the model, its value is factored in (1).

$\mathrm{VO}_{2}=3.5+0.1$ (speed +1.8 (speed)(incline)

...(1)

The metabolic estimation $\mathrm{VO}_{2}$ is mapped to caloric consumption via Equation (2).

$E=\left(\mathrm{VO}_{2}-B M R\right) * 5 /$ (Body_Mass) ...(2)

\footnotetext{
$\frac{\frac{5}{3}}{\frac{2}{0}}$

The statistical analysis proves that:

- Gravity Y linearly correlates to surface incline.

- Gravity Y values differ significantly between different surface inclines. 